\title{
Engineering and Informatics Student Multidisciplinary Learning using 3D Visualization and 3D Display of Radio Frequency (RF) Concepts
}

\author{
Lauren Christopher \\ Indiana University / Purdue \\ University at Indianapolis \\ Indianapolis, USA \\ lauchris@iupui.edu \\ Anthony Chase \\ Indiana University / Purdue \\ University at Indianapolis \\ Indianapolis, USA \\ chaseam@iu.edu
}

\author{
Albert William \\ Indiana University / Purdue \\ University at Indianapolis \\ Indianapolis, USA \\ almwilli@iupui.edu \\ Mihir Piyush Joshi \\ Indiana University / Purdue \\ University at Indianapolis \\ Indianapolis, USA \\ mipjoshi@umail.iu.edu
}

\author{
Anusha S. Rao \\ Indiana University / Purdue \\ University at Indianapolis \\ Indianapolis, USA \\ asathyan@iupui.edu \\ Wendy Krogg \\ Indiana University / Purdue \\ University at Indianapolis \\ Indianapolis, USA \\ wproud@umail.iu.edu
}

\author{
Ashley Dale \\ Indiana University / Purdue \\ University at Indianapolis \\ Indianapolis, USA \\ daleas@imail.iu.edu
Bree Abernathy
University at Indianapolis
Indianapolis, USA
baaberna@umail.iu.edu \\ Indiana University / Purdue
}

\begin{abstract}
This full paper addresses the Innovative Practice Category. We discuss our multidisciplinary approach to create a truly 3D representation and 3D display of $R F$ signals in space through the development of two different training tools to enhance student understanding of Radio Communications. Both tools show the data on 3D autostereoscopic displays rather than rendered back to $2 \mathrm{D}$ displays. The first new tool is a series of $3 \mathrm{D}$ stereoscopic animations created by a multidisciplinary team of students from the Media Arts and Sciences (School of Informatics) and Electrical Engineering (School of Engineering) programs for use with an autostereoscopic display, where each animation focuses on a single topic within RF communication learning, using real-world examples. The second innovative tool models the Navy use-case of Electronic Warfare (EW) using examples with 3D antenna radiation patterns of signal propagation using U.S. Navy's SIMDIS interactive 3D visualization environment. The developed scenarios are displayed on an autostereoscopic display, allowing students to manipulate RF signals in a 3D environment. Learning gains were assessed via a $2 \times 2$ crossover experimental design an engineering student group. Compared to the control group, students showed gains in understanding of the 3D shape of dipole antennas and understanding of the multiple RF antennas in a cell phone, and the connections between mobile phone antennas and cell towers. The results from these interventions collectively indicate that a truly 3D representation in space can be used to enhance students' understanding of antennas and RF signals.
\end{abstract}

Keywords- multidisciplinary training, $3 D R F$ antenna modeling, $3 D$ display, interactive $3 D$ visualization

\section{INTRODUCTION}

The importance of a solid undergraduate foundation in electromagnetic field (EMF) and radio frequency (RF) theory has been well documented [1] [2]. Furthermore, the abstraction and difficulty of understanding these topics continues to motivate research into how instruction methods can be improved, particularly through the use of technology in a

This research is sponsored by the US Navy (Office of Naval

Research) grant N00014-16-1-2810, with the support of NSWC-

Crane Division in Crane, India classroom and laboratory setting [3] [4] [5]. Recent work to improve EMF and RF instruction by researchers such as Xue [4] and Nguyen et al. [6] incorporate 3D visualization of signals and fields in undergraduate-level antenna design and propagation theory instruction. When done well, 3D data visualization has shortened training times, improved training recall, and improved task performance (speed, accuracy). For example, research has shown 3D viewing significantly improves surgical training and performance [7] [8] [9] [10]. Although Xue and Nguyen et al. both implement 3D models of antenna radiation patterns in their work, ultimately these patterns continue to be presented in a 2D format behind a screen. In this paper we discuss our approach to create a 3D representation of RF signals in space through the development of two stereoscopic training tools to enhance student understanding.

The first tool is a series of 3D short film animations for use with an autostereoscopic display, where each short film focuses on a single topic within RF communication concepts, then places these topics in an applied, real-world setting. The second tool consists of 3D antenna radiation patterns imported into the U.S. Navy's SIMDIS [11] interactive visualization environment then displayed on the autostereoscopic display, allowing students to manipulate RF signals in a $3 \mathrm{D}$ environment for increased understanding.

Two student groups were assessed, the Electrical and Computer Engineering Electric and Magnetic Fields (ECE311) class, and the ECE-Informatics multidisciplinary Stereoscopic Production and Display (N444). For the multidisciplinary course, results from the pre-post surveys show positive gains in terms of perceived alignment between their own course work and research in the United States Navy. The learning outcomes from the collaborative projects indicated that the students had an improved awareness of the RF signals and antennas, and were able to design 3D models to visualize them. For the engineering course on Electric and Magnetic fields, 
students completed pre-post knowledge questionnaire on RF signals and antenna concepts, based on the first new tool (3D animations). These students showed increased understanding of dipole antenna shapes and higher order learning after the 3D animation viewing.

\section{3D ANIMATION LEARNING TOOL}

\section{A. Three Dimensional Modeling of Antenna Radiation Patterns}

The MATLAB R2016a Antenna Toolbox was used to design antennas, then calculate the Electro-Magnetic Field (EMF) of the antenna in spherical coordinates rather than the traditional two-dimensional polar plots depicting the antenna profile. This holistic method of evaluating the entire radiation pattern rather than 2D slices is fundamental in creating 3D visualizations of RF signals. Examples of EMF calculations include the normalized power-radiation pattern by a half-wavelength dipole antenna and a six-element Yagi-Uda antenna.

A MATLAB script then converted the numerical field calculations into a 3D model of the EMF pattern and exported the model as a .obj (3D object) file suitable for other platforms. Model detail was controlled by varying the polygon count in the file, which in turn was controlled by varying the incremental sweep of azimuth and elevation calculations during the MATLAB EMF simulation. The file of the EMF was then imported into animation software packages such as Autodesk Maya 2017 for further optimization and texturing. This completed preparation of the .obj for use in animations and visualization platforms.

\section{B. Stereoscopic Animation and Display}

Six topical short-films were created to explain fundamental RF signal concepts: Theory and Function of a Dipole Antenna, Antenna Radiation Patterns, Communicating With Antennas, Antenna Signal Propagation, Digital Signals from Antennas, and Mobile Phone Radiation Patterns. Although these topics are closely related, the films are structured for independent viewing. Each film was created using stereoscopic techniques that place a visualization of the MATLAB-generated 3D pattern in space, and an accompanying narration provides added exposition. For example, changes in antenna design are linked to changes in RF signal directivity, RF interference is shown affecting 3D RF signals, etc.

The final 3D animations are formatted for an autostereoscopic display to allow for group viewing without the use of 3D glasses or head-mounted displays. By using an autostereoscopic display, we circumvent documented issues with eyestrain when viewing stereoscopic material [11] [12], making the films equally accessible to all viewers.

The data for the autostereoscopic display is shown in Figure 1 with the left side of the image the 2D color, and the right side of the image is the depth map for 3D viewing. The whiter areas

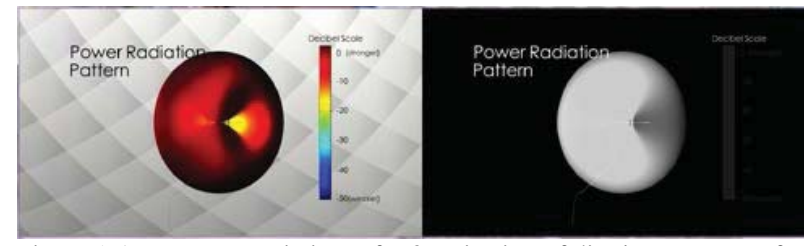

Figure 1 Autostereoscopic input for 3D viewing of dipole antenna. Left: 2D color image. Right: depth map for input to autostereo display.
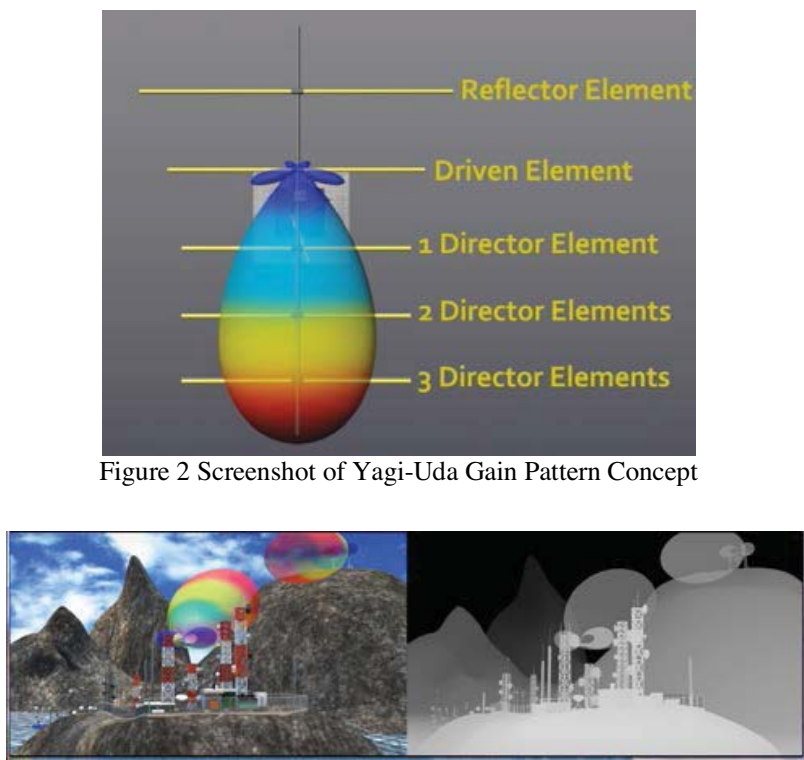

Figure 3 Screenshot showing Path Loss and Communication Concepts. Left: 2D color image. Right: depth map on right for input to autostereo display.

of the depth map will appear closer to the viewer in the final display.

\section{Learning Goals for Animations}

Chapter 1: Theory and Function of a Dipole Antenna Learning goals: 1- Introduction to a dipole antenna and its relationship between antenna length and transmission frequency, 2-3D toroid shape of the antenna gain in Figure 1.

Chapter 2: Antenna Radiation Patterns - Learning goals: 1- Introduction to various antenna shapes and how these relate to the resulting antenna gain pattern. 2- Understanding the stepby-step process to create a Yagi-Uda antenna pattern shown in Figure 2 .

Chapter 3: Communicating With Antennas - Learning goals: 1- Understanding the concept of nulls and lobes in the gain of antennas, 2- Understand the power level transmitted by the antenna, and that it is possible to steer the main beam such that interference falls within a null in the pattern, 3- The example in Figure 3 is used to teach the idea of far-field signal path and directionality of the Yagi-Uda antenna gain pattern.

Chapter 4: Antenna Signal Propagation - Learning goals: 1- Student can discuss various ways that RF signals are propagated, such as sky wave, surface wave, and line of sight. 2- The example in Figure 6 shows the sky wave. 
Chapter 5: Digital Signals from Antennas - Learning goals: 1- Show understanding of digital transmission of signals, including modulations such as AM, FM PSK and QAM. 2Understanding the basic concepts of Forward Error Correction (FEC).

Chapter 6: Mobile Phone Radiation Patterns - Learning goals: 1- Understand the function and breakdown of the number of antennas in a typical smartphone. 2- Show the ability of an attacker to sense the radiation pattern of a smartphone.

\section{3D INTERACTIVE LEARNING TOOL}

\section{A. Using Antenna Models in SIMDIS}

SIMDIS is a data visualization platform developed by the US Naval Research Laboratory, and enables simulation and analysis for 2-D and 3-D visual content [11]. This visual content can be a live-feed linked to SIMDIS via APIs and then displayed, or it can also be a post-processed simulation result. Figure 4 demonstrates the environmental capabilities of SIMDIS for use when demonstrating RF signals in an applied context.

In this project SIMDIS is used to develop Electronic Warfare use-cases. This involves importing the 3D MATLAB antenna radiation patterns after they have been refined in Autodesk Maya to depict and help a SIMDIS user understand RF concepts. These scenarios allow warfighters to interact with RF signals by controlling their location, direction, and power; without the need to calculate the underlying RF physics instantaneously. Examples of interactive scenarios include portraying ship-toshore communications, sky-wave transmissions, and antennabeam steering.

To create an interactive scenario, first a SIMDIS file (.asi) needs to be written. This file contains all the necessary data including an object's Time Space Positional Information (TSPI), coordinate systems e.g. LLA (Latitude, Longitude and Azimuth), timing sequence, velocity, roll, pitch, and yaw. After specifying these variables, the end user is able to view and interact with the scenario.

SIMDIS scenarios were created to complement each 3D animated short-film, except for Chapter 5, and depict RF signal propagation in a Navy use-case. These SIMDIS scenarios naturally exist in an interactive format, but a demonstration of each scenario is also recorded for group viewing on the autostereoscopic display. Figure 5 is a screen-shot from the SIMDIS interactive scenario depicting how two RF signals must overlap in time and space as a pre-requisite for communication. In Figure 5, the RF signals are depicted as originating from a dipole antenna on a helicopter, and a Yagi-Uda antenna onboard a ship. These patterns were chosen for their visual distinctiveness rather than factual implementation, with the goal of helping viewers visualize different 3D patterns interacting in space.

Figure 6 is a screenshot depicting sky-wave signal propagation in SIMDIS, where an antenna in Chicago is capable of transmitting beyond its regular range to a receiving antenna in New York City, reflecting off of the ionosphere (blue band).

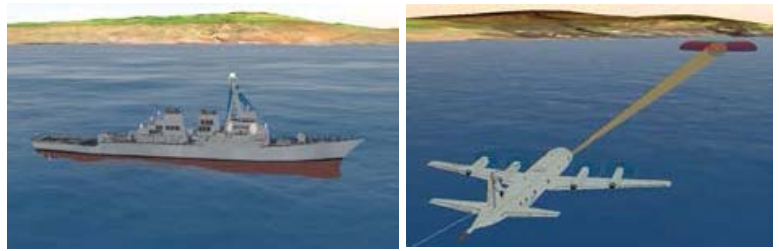

Figure 4 SIMDIS Visualization examples

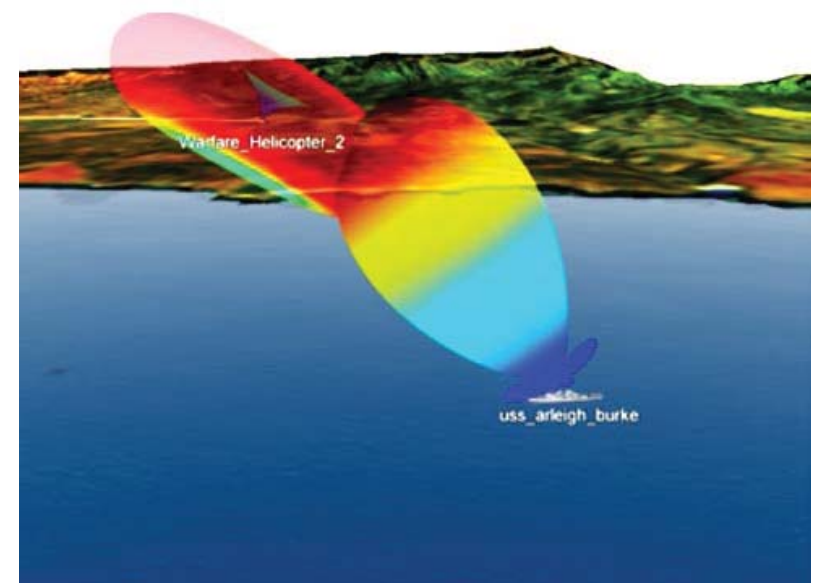

Figure 5 Example of 3D antenna radiation patterns imported from MATLAB. color indicates antenna gain.

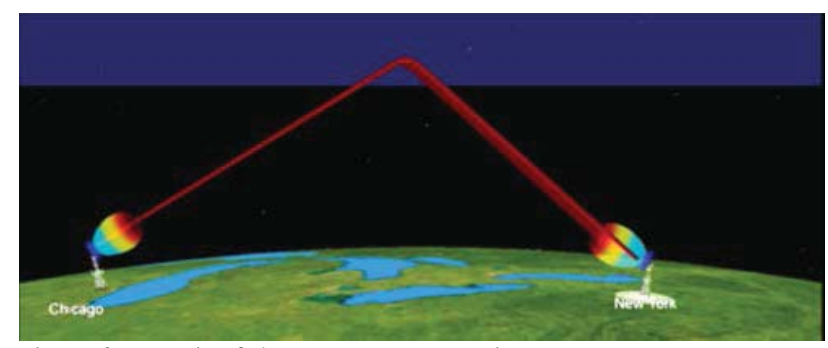

Figure 6 Example of sky-wave RF propagation

\section{B. Learning Goals for Interactive Environment}

Chapter 1: Theory and Function of a Dipole Antenna Learning goals: 1 . demonstrate a movable ship and respective antenna patterns in $3 \mathrm{D}$ visualization.

Chapter 2: Antenna Radiation Patterns - Learning goals: demonstrate a ship with a Yagi-Uda antenna gain pattern.

Chapter 3: Communicating With Antennas - Learning goals: demonstrate the concept of directionality by pointing of the antenna gain to place the unwanted enemy signal in the null of the Yagi-Uda antenna.

Chapter 4: Antenna Signal Propagation - Learning goals: demonstrate student's understanding of sky wave to show propagation and skip zones.

Chapter 5: Digital Signals from Antennas - Learning goals: no interactive component in this chapter.

Chapter 6: Mobile Phone Radiation Patterns - Learning goals: demonstrate how a radiation pattern from a mobile phone on a ship may be sensed by an airplane's sensor.

When the interactive SIMDIS element is implemented with the $3 \mathrm{D}$ short-film animations, the result is a simplified, 3D 
overview of antenna theory and propagation entirely in autostereoscopic format, where the films form a lecture component and the SIMDIS scenarios a virtual laboratory.

\section{Additional Benefits with $3 D$}

One side benefit to producing $3 \mathrm{D}$ graphics models is that these models are available for use in $3 \mathrm{D}$ printing. A 3D print of some of the simpler models is seen in Figure 7. This element of learning has not yet been tested. A 3D tactile experience may be useful for some learners.

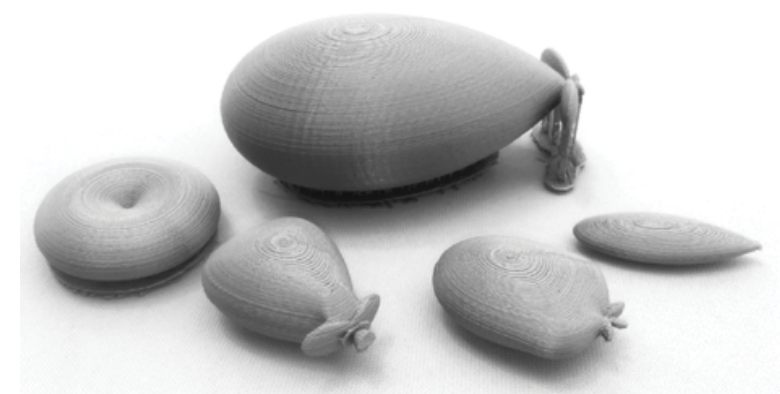

Figure 7 3D printed antenna models, top: Yagi-Uda: bottom: Dipole, Vivaldi, Waveguide, and Horn.

\section{RESULTS}

\section{A. Engineering student learning}

The 32 engineering students in the Electrical and Computer Engineering ECE311 Electromagnetics class were studied after 10 weeks in the course when they had already been introduced to some antenna concepts. The class was divided into two random groups to support a $2 \times 2$ crossover experimental design for comparison of control and treatment. The identifying variable for consideration in this case is the topics of the animations. Each group completed a pre and post questionnaire assessing understanding of content. Each question number in the survey corresponded to a learning goal in the same chapter number. Performance was then assessed in relation to the topics that corresponded to the videos they watched, and results are shown in Table 2. This crossover design is displayed in Table 1 below.

\begin{tabular}{|c|c|c|c|}
\multicolumn{4}{|c|}{ N444 Course Students } \\
\hline \multirow{2}{*}{ Group } & $\begin{array}{c}\text { Treatment } \\
\text { Topic }\end{array}$ & $\begin{array}{c}\text { Control } \\
\text { Questions }\end{array}$ & $\begin{array}{c}\text { Treatment } \\
\text { Questions }\end{array}$ \\
\hline 1 & $\begin{array}{c}\text { FILL IN } \\
\text { TOPIC }\end{array}$ & $1,3,5$ & $2,4,6$ \\
\hline 2 & $\begin{array}{c}\text { FILL IN } \\
\text { TOPIC }\end{array}$ & $2,4,6$ & $1,3,5$ \\
\hline
\end{tabular}

Students showed gains in understanding of the 3D shape of dipole antennas (question 1, yellow highlight), the understanding of digital transmission (question 5, gray highlight), and the understanding of the multiple RF antennas in a cell phone (question 6 , blue highlight). The digital transmission and the connections between mobile phone antennas and cell towers represent some of the more difficult concepts in the course. For example, question 5 and 6 specifically are not textbook material and demonstrates the possibility of higher order learning such as application of concepts related to the treatment.

\begin{tabular}{|c|c|c|c|}
\hline \multicolumn{4}{|c|}{$\begin{array}{l}\text { GROUP 1: } n=14 \text { students } \\
\text { Treatment animations: \#1, 3, and } 5\end{array}$} \\
\hline Question & pre-score & $\begin{array}{l}\text { post- } \\
\text { score }\end{array}$ & $\begin{array}{l}\% \text { gain }=(\% \text { post }- \\
\% \text { pre }) / \% \text { pre }\end{array}$ \\
\hline 1 & $21 \%$ & $50 \%$ & $29 \%$ \\
\hline 2 & $21 \%$ & $57 \%$ & $36 \%$ \\
\hline 3 & $71 \%$ & $64 \%$ & $-7 \%$ \\
\hline 4 & $43 \%$ & $86 \%$ & $43 \%$ \\
\hline 5 & $60 \%$ & $98 \%$ & $38 \%$ \\
\hline 6 & $43 \%$ & $43 \%$ & $0 \%$ \\
\hline \multicolumn{4}{|c|}{$\begin{array}{l}\text { GROUP } 2: n=18 \text { students } \\
\text { eatment animations: \#2, 4, and } 6\end{array}$} \\
\hline Question & Pre score & $\begin{array}{l}\text { Post } \\
\text { score }\end{array}$ & $\begin{array}{l}\% \text { gain }=(\% \text { post }- \\
\% \text { pre }) / \% \text { pre }\end{array}$ \\
\hline 1 & $67 \%$ & $89 \%$ & $22 \%$ \\
\hline 2 & $44 \%$ & $67 \%$ & $22 \%$ \\
\hline 3 & $72 \%$ & $89 \%$ & $17 \%$ \\
\hline 4 & $50 \%$ & $83 \%$ & $33 \%$ \\
\hline 5 & $44 \%$ & $56 \%$ & $11 \%$ \\
\hline 6 & $17 \%$ & $69 \%$ & $53 \%$ \\
\hline
\end{tabular}

Table 2. Learning Gains from ECE Student Treatment Groups

\section{B. Multidisciplinary student learning}

The N444 multidisciplinary course had 6 students, with one engineering student, and 5 Media Arts students. These students had a lecture-style presentation about antenna and RF first, followed by a pre-test before the animations and interactive tool, and a post-test after the animations and interactive tool. The results from the content-based test in Table 3 show a gain in many of the items. Some items display small gains and others no gain at all. We believe this is due to a ceiling effect in which the pre scores were very high and left little room for improvement after the intervention.

\begin{tabular}{|c|c|c|c|}
\multicolumn{4}{|c|}{ N444: $\mathbf{n = 6}$ students } \\
\hline \multirow{2}{*}{$\begin{array}{l}\text { Question } \\
\text { score }\end{array}$} & $\begin{array}{l}\text { Post } \\
\text { score }\end{array}$ & $\begin{array}{l}\text { \% gain = (\%post - } \\
\% \text { pre)/\%pre }\end{array}$ \\
\hline 1 & $83 \%$ & $100 \%$ & $17 \%$ \\
\hline 2 & $100 \%$ & $100 \%$ & $0 \%$ \\
\hline 3 & $67 \%$ & $83 \%$ & $17 \%$ \\
\hline 4 & $67 \%$ & $83 \%$ & $17 \%$ \\
\hline 5 & $83 \%$ & $83 \%$ & $0 \%$ \\
\hline 6 & $67 \%$ & $100 \%$ & $33 \%$ \\
\hline
\end{tabular}

Table 1. Learning Gains from N444 students 


\section{CONCLUSION AND FUTURE WORK}

The results from these interventions collectively indicate that a truly $3 \mathrm{D}$ representation in space can be used to enhance student understanding of antennas and RF signals. Survey instruments in both courses indicate that there were gains in content knowledge across both courses that may be attributed to the intervention of the animations. These percentage gains indicate the future need for specific testing regarding the aspects of the animations or possible attitudinal impact that they may have had. The results from this study contain a maturation threat to internal validity. The results are promising to the use of these activities to help students understanding. There is a possibility, however that some other experience that the students received outside of this class impacted the observed measurements.

Future work will be to repeat the testing with another cohort of ECE311 students in Fall 2018, continue to refine the animation 3D videos and the SIMDIS interactive learning, and repeat the testing with Spring 2019 N444 class. We also plan to include testing of students for retention of knowledge by testing them again some weeks after initial post-survey. Validation of the knowledge questionnaire will also strengthen learning gains obtained from this intervention.

\section{ACKNOWLEDGMENT}

The funding from ONR-STEM and direction from NavyCRANE employees (Dr. Marble, Mr. Zipperle, Mr. Seyer) were vital to this research. The authors would also like to acknowledge the contributions of additional Media Arts students who were key to developing the animations: Bryan Keefe, Mia Sulaiman, Megan Hallam, Brandon Raleigh, Jacob McCarthy and Patrick Bellido-Choy.

\section{REFERENCES}

[1] K. Gupta, T. Itoh and A. A. Oliner, "Microwave and RF education past, present, and future," IEEE Transactions on Microwave Theory and Techniques, vol. 50, no. 3, pp. 1006-1014, 2002.

[2] K. Keltikangas and H. Wallen, "Electrical Engineer's perceptions on education - electromagnetic field theory and its connection to working life," European Journal of Engineering Education, vol. 35, no. 5, pp. 479-487, 2010.

[3] M. Popovic and D. Giannacopoulos, "Giving life to teaching intorductory electromagnetics: a three-year assessment plan," in Antennas and Propagation Society International Symposium, 2004.

[4] J. Xue, "Web-based interactive 3D electronmeagnetic visualization for antenna designs," in Antennas and Propagation Society international Symposium (APSURSI), 2014 IEEE, 2014.

[5] P. Crilly, "An innovative approach to teaching an undergraduate electromagnetics, antennas and propagation course," in American Society for Engineering Education, Zone 1 Conference of the IEEE, 2014.

[6] D. H. Nguyen, L. Henderson, J. Chacko, C. Sahin, A. Paatelma, H. Saarnisaari, N. Kandasamy and K. R. Dandikar, "Beamviewer: visualization of dynamic antenna radiation patterns using augmented reality," in Computer Communications Workshops (INFOCOM WKSHPS), IEEE Conference on, 2016.

[7] N. Taffinder, S. G. Smith, J. Huber, R. C. Russell and A. Darzi, "The effect of a second-generation $3 \mathrm{~d}$ endoscope on the laparoscopic precision of novices and experienced surgeons," Surgical Endoscopy, vol. 13, no. 11, pp. 1087-1092, 1999.

[8] O. Wasserzug, N. Margalit, N. Weizman, D. Fliss and Z. Gil, "Utility of a three-dimensional endoscopic system in skull base surgery," Skull Base, vol. 20, no. 4, 2010.

[9] R. P. Manes, S. Barnett and P. S. Batra, "Utility of novel 3-dimensional stereoscopic vision system for endoscopic sinonasal and skull-base surgery," International Forum of Allergy \& Rhinology, vol. 1, no. 3, pp. 191-197, May/June 2011.

[10] J. C. Byrn, S. Schluender, C. M. Divino, J. Conrad, B. Gurland, E. Shlasko and A. Szold, "Three-dimensional imaging improves surgical performance for both novice and experienced operators using the da Vinci robot system," American Journal of Surgery, vol. 193, no. 4, pp. 519-522, 2007.

[11] "SIMDIS User Manual," 28 March 2017. [Online]. Available: https://simdis.nrl.navy.mil/doc/SIMDIS.aspx. [Accessed 23 April 2018].

[12] H. Heo, W. O. Lee, K. Y. Shin and K. R. Park, "Quantitative measurement of eyestrain on 3D stereoscopic display considering the eye foveation model and edge information," Sensors, vol. 14, no. 5, pp. 8577-8604, 2014.

[13] J. C. Read and I. Bohr, "User experience while viewing stereoscopic 3D television," Ergonomics, vol. 57, no. 8, pp. 1140-1153, 2014. 